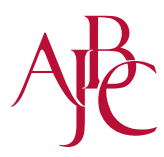

\title{
Holistic View on Scientific Skin-whitening
}

Zhuo Du, Li Li, Yinmao Dong*

China Cosmetic Collaborative Innovation Center, Beijing Technology and Business University, Beijing, China

*Corresponding author: Yinmao Dong, China Cosmetic Collaborative Innovation Center, Beijing Technology and Business University, No. 11/33, Fucheng Road, Haidian District, Beijing, China Tel.: +861068984937

Email: ymdong2008@163.com

Received February 25, 2016

Revised May 30, 2016

Accepted June 9, 2016

Published June 30, 2016

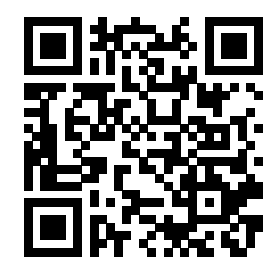

\begin{abstract}
Purpose: The aim of this paper is to comprehensively analyze the related factors affecting skin color and provide new ways for healthy whitening. Method: Firstly, we discussed the root factors affecting skin color and in detail reviewed several exploitable ways which can achieve scientific skin-whitening, Finally, we analyzed three aspects from the mechanism level: melanin metabolism, blood circulation and dermal collagen fibers, which we hoped to develop new ways for whitening cosmetic additives. Result: We can open up new ways of inhibiting keratinocyte cells releasing related cytokines, promoting the blood circulation and inhibiting the fracturing of dermal collagen fiber to achieve healthy whitening. Conclusion: Skin whitening has close associations with the optical properties of the skin. Each layer of the skin can affect the skin color, we should follow the rules of skin metabolism, to achieve skin whitening in scientific ways.
\end{abstract}

Keywords: Holistic view, Skin-whitening, Optical property, Blood circulation, Collagen fibers

\section{Introduction}

整体就是统一性和完整性。整体观是指从全局考虑问题的观 念。随着社会的进步、科技水平的提升，科技工作者往往对 某一个靶点问题研究得科学且深入, 取得了一个又一个的辉煌 成果。然而, 将这些成果转化利用时, 往往会出现极端地强 化某一点而忽略整体的现象。例如, 在美容化妆品领域美白 的相关研究和应用中就出现了因过度强调抑制酪氨酸酶活 性、抑制黑素产生, 而导致了一系列安全事件。本文中, 笔 者从整体观出发, 全面科学地理解皮肤美白, 旨在能给美白功 效原料的开发提供新思路。

\section{From the visual aspect to interpret skin whitening}

我们感受到物体的形状、大小、颜色、质地等都是通过人眼 接收物体反射的可见光(400nm-700nm), 进而在大脑皮层产生
的主观感觉。感受肤色亦是如此。外界光源照射到皮肤上, 与 皮肤发生界面反射、散射、本体反射、吸收等相互作用, 外界 光源经过这些作用后转化成有色光, 进入眼, 从而形成人们对 肤色的判断。

人体皮肤分为三层: 表皮、真皮、皮下组织。光线照射到皮肤 上, 先发生界面反射 (4\%-8\%), 其余光线透射进入皮肤, 在表皮 和真皮等皮肤层次中被吸收和散射, 散射光中的一部分溢出表皮形 成皮肤的本体反射。表皮主要起吸收光线作用, 其散射作用可忽略 不计, 其吸收作用主要与其基底层黑素细胞分泌的黑素的含量、 分布和类型有关。其中黑素包括优黑素和褐黑素, 分别呈褐色和 黄至红褐色, 吸收光谱较宽, 能及收整个可见光和紫外光(Chen \& Liu, 2006)。光线透过表皮, 进入真皮, 真皮主要对光线进行散射 和吸收, 真皮中能吸收光线的载色体为血液中的血红蛋白、胆红 素和类胡夢卜素等成分, 光线遇到真皮层中的胶原蛋白会发生本 体反射。由此可见, 肤色与皮肤结构的光学属性密切相关。 


\section{Factors affecting the skin-whitening and related mechanisms}

由上述可知, 皮肤的各层结构均能影响光线与皮肤的相互作 用, 进而影响人眼对肤色的判断。所以, 美白不仅仅只是黑素 的问题, 黑素、血红素、类胡夢卜素、胆红素、黄化的胶原纤 维等相关色素作为载色体能决定肤色, 其他因素例如皮脂膜状 态、角质层的厚度及含水量等也能影响肤色。故研究美白我 们要从整体出发, 从皮肤的整体观来分析美白问题, 遵循皮 肤代谢科学规律, 避免过分强化某一因素而引发各种安全问 题。下面即根据皮肤的结构针对能影响肤色的因素及相关的 美白路径做总结分析。

\section{The skin surface microecology}

皮肤表面微生态主要由常驻菌群 (如痤疮丙酸杆菌、表皮葡萄 球菌等) 和暂住菌群 (如棒杆菌、需氧革兰氏阴性杆菌及产色素 的微球菌等) 组成，构成了皮肤的微生物屏障。当皮肤表面微生 态失衡时即会出现多种皮肤问题，例如产色素的微球菌大量繁 殖时，其产生的色素超过皮肤局部自净能力，使得过多的色素 在表皮沉积, 这些色素能作为载色体吸收光线, 形成黄褐斑。 故我们在研发美白化妆品时可通过调整皮肤微生态平衡进而 达到改善色斑的目的。

\section{The state of skin sebum membrane}

皮脂膜是位于皮肤表面, 由皮脂腺里分泌出来的皮脂、角质 细胞产生的脂质及从汗腺里分泌出来的汗液加空气中的灰尘、 细菌、病菌等等融合而形成的一层膜。角质层死细胞的反射是 无规律的, 皮肤表面的光泽主要由皮脂膜和覆盖在其上的化学 物质决定。除此之外，皮脂膜还能起到润泽皮肤的作用。故要 想有白晳透亮的肌肤, 拥有健康的皮脂膜很重要。皮脂膜健康 即为皮肤油水分泌平衡, 若油水分泌比例不平衡, 则会出现看 起来过油或过黯淡的现象。此外，健康的皮脂膜对维护皮肤的 屏障功能具有重要作用, 若皮脂膜受损, 则皮肤易出现敏感潮 红、红血丝等现象, 影响肤色。故美白化妆品的开发可通过调 节皮肤油水分泌平衡，改善皮脂膜状态，提升皮肤质感，进而 达到使肤色自然透亮的目的。

\section{Epidermis}

角质层、透明层、颗粒层、棘层为死细胞, 光线在其中主要 发生散射作用及遇到透明颗粒状物质则发生本体反射。有资 料显示, 这几层的薄厚亦能影响我们对肤色的判断, 例如, 角 质层厚, 肤色偏黄; 颗粒层和透明层厚, 肤色显白 (He, 2011)。 除了各层薄厚外, 各层的含水量也能很大程度上影响肤色, 若
皮肤缺水, 角质层易出现干燥、鳞屑状态, 使光线发生非镜面 反射而成灰暗状态, 若皮肤含水量充足, 则皮肤表面多发生规 则的反射而呈现明亮、有光泽状态。此外, 皮肤含水量多少对 角质细胞的功能有重要影响, 若皮肤缺水, 角质形成细胞功 能与结构障碍, 不能将黑素及时均匀地运输到表皮导致黑素代 谢异常 (Ramos-e-Silva \& Jacques, 2012), 同时, 皮肤缺水还 会导致皮肤屏障功能受损，导致皮肤易受外界刺激和紫外线影 响, 紫外线照射会导致酷氨酸酶和黑素相关蛋白激酶活性增 加 (Baroni et al., 2012), 对外界刺激敏感易使皮肤发生炎症 反应, 导致炎症后色素沉着。由此可见, 只有当皮肤含水量充 足, 薄厚适中时, 皮肤才会呈现柔软滋润、光滑细淢的状态。 故我们在美白的同时, 不要忽视保湿的重要性, 维持皮肤整体 的健康状态, 皮肤才会白晳透亮。

\section{Melanin metabolism}

在影响肤色的诸多因素当中, 对肤色起决定作用的为皮肤中 的能吸收光线的载色体, 包括有黑素、血红素、类胡夢卜素、 黄化胶原纤维等, 黑素为其中的主要因素。黑素由位于表皮基 底层的黑素细胞产生，黑素细胞大约占基底层细胞的10\%, 个黑素细胞通过树枝状的突起与周围10-36个角质形成细胞形 成表皮黑素单元，表皮黑素单元决定了肤色。

黑素分为优黑素和褐黑素。黑素在黑素细胞中的黑素小体中 产生后, 一部分会随树枝状突起转移至角质形成细胞, 并随表 皮细胞移行脱落排出 (Gillbro \& Olsson, 2011; Luet al., 2000), 一部分会下移至真皮层的毛细血管, 随血液循环由肾脏排出 (Yan et al., 2009)，未排出的部分，则沉着于皮肤中构成人 的肤色。正常情况下, 皮肤中进行着色素产生、排出、沉着 的平衡维持着人的固有肤色, 但受到外界因素影响时, 黑素 代谢的平衡被打破, 固有肤色发生变化, 会出现色素沉着过 度而使皮肤过黑或黑素细胞合成黑素、转运黑素的能力弱则 会导致白斑等现象。故维持黑素代谢平衡, 对维持健康的肤 色非常重要。在基础研究中, 关于黑素代谢方面的研究相当 多, 但在美白功效成分实际开发应用中, 大多数仍着眼于抑制 酪氨酸酶路径, 且过分强化该路径, 过度抑制酪氨酸酶活性, 而引发了一系列安全问题。故在通过黑素代谢路径开发美白功 效的添加剂时, 应注意整体观, 合理地把握度, 不要过分地破 坏皮肤本身的黑素代谢平衡。

\section{Blood microcirculation}

皮肤的真皮和皮下组织中含大量的毛细血管, 血管中血红蛋白 作为皮肤的载色体能影响肤色, 氧合血红蛋白多时, 皮肤呈鲜 红色, 脱氧血红蛋白多时, 皮肤呈暗紫红色。即真皮中毛细血 管的舒缩、数量、血流量和血红蛋白含氧量都能影响肤色。真 
皮层血管收缩痉挛或随着年龄增长, 皮肤中血管内皮生长因子 水平逐渐下降，皮肤毛细血管数量逐渐减少，皮肤会因血流量 减少而呈现苍白色 (Huang et al., 2013)。在某些疾病状态下, 由 于皮肤局部静脉迂曲，血液回流受阻，毛细血管压增高，红细 胞外漏, 含铁血黄素沉积而使皮肤呈黄褐色(Huang et al., 2013) ; 此外, 丰富的血流量, 能及时将细胞代谢产生的各种有害物 质等清除，为皮肤细胞提供充足的氧气和营养物质，使皮肤的 各种细胞维持良好功能，角质层水分充足，保持皮肤表面明亮 光泽。另外，有文献报道，黑素在黑素细胞中合成后，一部分 随角质形成细胞向上迁移至剥脱排出, 另一部分会下移至真皮 中的毛细血管，随血液循环通过肾脏排出，所以，加强皮肤血 液微循环亦能加快黑素消散, 进而起到美白的功效 (Yue et al., 2011)。故我们在研发美白功效添加剂时需兼顾改善皮肤微循环 部分，从而能更好的改善皮肤颜色。

\section{The state of skin consenescence}

肌肤表面老化会导致肤色泛黄, 没有光泽, 它是亚洲中老年 女性最为常见的生理衰老现象, 并且随着年龄的增大而愈发显 得严重, 呈现出人们常说的面色暗黄 (Gu et al., 2011)。皮肤衰 老过程中产生的代谢垃圾堆积于皮肤中, 能作为吸收光线的载 色体影响肤色。

皮肤衰老过程中，机体内自由基代谢处于不平衡状态，过量的 自由基可将不饱和脂肪酸氧化成丙二醛，丙二醛是很强的交联 剂，与蛋白质、核酸等大分子交联，可形成难溶性脂裼素，使 肤色暗黄 (Fang \& Zhao, 2010)。另外，体内会发生非酶糖基化作 用，即无酶催化的条件下，还原性糖的醛基或酮基与蛋白质等 大分子中的游离氨基反应生成可逆或不可逆结合物 一一 高级 糖基化终末产物 (advanced glycation end-products, AGEs) 的过 程。皮肤真皮层中富含胶原蛋白和弹性蛋白，蛋白质分子中的 氨基酸易与细胞外液中葡萄糖醛基或酮基发生反应生成可逆 或不可逆结合物一 变后形成棕黄色垃圾, 堆积于皮肤真皮层, 使肌肤失去剔透感而 泛黄、暗沉 (Lai et al., 2009)。由此可见，我们可通过清除自由 基、抗氧化、抑制非酶糖基化等过程, 解决因皮肤衰老带来的 肤色暗黄问题。

\section{Several science problems to be studied}

传统美白剂主要通过抑制黑色素细胞增殖、抑制酪氨酸酶、加 速角质层脱落等方式最终起到干预黑素的形成与代谢来发挥美 白功效。这种途径虽然能够达到一定的美白效果，但过度干预 黑素代谢会一方面破坏了黑素对人体的保护作用，另一方面会
破坏皮肤固有肤色，导致 “白斑病” 等。所以，目前国内外研 究逐步关注在不干预黑素正常代谢的情况下寻找健康、安全的 美白活性物质, 例如通过促进色素分布均匀、促进血液循环、 清除及减少皮肤垃圾、抑制炎性因子释放等多种途径达到美白 的效果。本文中, 主要探讨三种路径, 旨在能为美白功效添加 剂的开发提供新思路。

\section{Reduced melanin deposition by inhibiting keratinocyte cytokine release}

黑素细胞位于表皮基底层，黑素细胞合成黑素，通过树突状 的突起将黑素颗粒转运到与之相联系的角质形成细胞, 而使 皮肤维持正常肤色。同时，角质形成细胞通过旁分泌释放细 胞因子影响黑素细胞 (Abdel, 1999; Gordon et al., 1989)。角质 形成细胞衍生的调节黑素细胞的细胞因子包括: 白细胞介素-1 (interleukin, LL-1)、白细胞介素-6(interleukin, L-6)、肿瘤不死因子 -a (tumornecrosis factor, TNF- $\alpha$ ), 内皮素-1 (endothelin, ET-1), 碱性成纤维细胞生长因子 (basic fibroblast growth factor, bFGF), 干扰素- $\gamma$ (interferon, IFN - $\gamma$ ), 转化生长因子- $\alpha$ (transforming growth factor, TGF - $\alpha$ ) 和 $\alpha$-促黑素细胞生长激素 (melanocyte stimulating hormone, $a-M S H)$ 。这些因子通过与黑素细胞表面相 应受体结合, 刺激胞内信号通路, 介导黑素代谢。

正常情况下, 黑素细胞受胞外细胞因子的调节, 维持黑素正 常代谢过程。但外界刺激会导致相关细胞因子上调, 促进黑 素合成增加及黑素细胞形态的变化等。例如, 有研究表明, 紫外线照射到皮肤上, 会导致皮肤发生炎症损伤。在UVB介 导的炎症反应中, 角质形成细胞核中的转录因子NFkB 被激 活, 进一步介导角质形成细胞分泌 TNF- $a 、 I L-1 、 I L-6$ 、前列腺 素E2 (prostaglandin E2, PGE2) 等炎症因子 (Ha et al., 2011)。 这些炎症因子能使ET-1 和膜结合干细胞因子 (mSCF) 的分泌上 调 (Niwano et al., 2015), 上调的ET-1和mSCF能分别与黑素细 胞上相应受体结合, 激活胞内信号通路, 刺激黑素细胞合成 黑素。传统的美白剂多关注于通过抑制黑素细胞内影响黑 素合成的关键通路从而达到抑制黑素合成的作用。例如通 过目前应用普遍的抑制酪氨酸酶活性、通过抑制相关受体 的表达 (Kasamatsu et al., 2014)、抑制黑素细胞胞内Ca2+流动 性 (Kobayashi et al., 2002)、抑制MEK/ERK1/2/RSK/CREB的 磷酸化 (Kim et al., 2015)、抑制microphthalmia associated transcription factor (MITF)、 tyrosinase related protein-1 (TRP-1) TYR-2等表达 (Wang et al., 2014)等。这些物质虽然具有明显的美 白功效, 但存在一定的弊端, 例如可能会出现过度干扰黑素 正常代谢，影响皮肤“固有肤色”引发“白斑病”等症状; 而 且, 美白活性成分需进入黑素细胞内才能达到胞内抑制的效 果, 对活性成分的透皮吸收有了更高的要求。所以, 如何在不 
干预黑素正常代谢的情况下达到健康、安全的美白目的值得被 大家关注和思考。针对这一问题, 我们提出, 可通过抑制角质 形成细胞释放相关细胞因子的胞外抑制方式, 而不干扰黑素细 胞胞内正常代谢过程，达到美白效果。目前关于胞外抑制有少 量研究，例如Ha et al. (2011) 研究表明金钱薄荷可能通过抑制 角质形成细胞对前炎症因子和黑素相关旁分泌因子的释放而不 是通过对黑素细胞中黑素合成活性进行抑制，起到美白效果; Parket al. (2008) 研究表明明日叶的已烷和乙酸乙酯馏分可通 过抑制角质形成细胞对UVB介导的ET-1的释放量来达到美白目 的; Kimet al. (2007) 研究表明短果茴芹既通过抑制酪氨酸酶 活性、酪氨酸酶、TRP-1、TRP-2等相关蛋白的表达，又通过能 减少ET-1细胞因子的释放量来达到美白的目的; Kobayashi et al. (2002) 研究表明蜀葵属植物提取物既能通过减少人角质 形成细胞 (normal human keratinocytes, NHKC) 对ET-1的释放 量, 也能抑制ET-1引起的 $C a^{2}+$ 流动性, 达到美白功效。

虽然关于胞外抑制有所研究, 但关于美白作用路径上, 目前国 内外研究重心仍偏向于胞内抑制方面, 且关于胞外抑制方面, 所 篮选出的活性物质, 除了具有抑制角质形成细胞释放相关细胞 因子外, 对黑素细胞胞内黑素代谢仍具较强抑制活性。所以, 我们认为, 仅通过胞外抑制角质形成细胞释放相关细胞因子, 但不影响黑素细胞胞内正常代谢过程的美白新路径具有很大的 研究价值, 且有潜力成为日后美白功效添加剂发展新趋势。

\section{Prevent the phenomenon of dark yellow skin color by inhibiting the fracture of collagen fibers}

真皮层中胶原纤维的降解通过三种方式影响肤色: (1)影响光 线在真皮层中的散射; (2)影响光线在皮肤表面的界面反射作 用; (3)胶原纤维降解后形成代谢垃圾。胶原纤维的降解与成纤 维细胞密切相关, 成纤维细胞可以分泌基质金属蛋白酶对胶原 纤维等进行降解。

基质金属蛋白酶 (metalloproteinases, MMPS) 是降解细胞外基质 (extracellular matrix, ECM) 的一类重要的蛋白水解酶类。其中, MMP-1可降解, I, I,II型胶原蛋白。MMP-3可以降解II, III, IV, IX, 和X型 胶原蛋白、蛋白聚糖、纤连蛋白、层粘连蛋白、弹性蛋白。 紫外线刺激下, 皮肤成纤维细胞内会发生一系列氧化还原反 应, 产生大量自由基(reactive oxygen species, ROS), ROS通过 刺激MAPK信号通路从而刺激成纤维细胞产生MMPS。其机制 为: 自由基与细胞内膜接触后, 膜表面受体磷酸化增加, 从 而激活MAPK信号通路中的C-Jun N末端蛋白激酶, C-Jun N末端 蛋白激酶激活后致使激活蛋白-1 (activator protein-1, AP-1) 被激 活, AP-1是细胞内与MMP-1密切相关的一个转录激活因子, 上 调MMP-1的表达。MMPs在一般状态下会有与基质金属蛋白酶 抑制剂 (tissue inhibitors of metalloproteinases, TIMPS) 结合, 处
于静息状态。但当受ROS刺激时, MMPS会与TIMPS形成复杂的 结构, MMPS被激活离开细胞进入细胞间质, 降解胶原蛋白。故 我们提出, 可通过抑制MMPs的产生抑制胶原蛋白的降解, 提高 皮肤的色泽度, 达到美白效果。

\section{Achieve a healthy skin color by promoting blood circulation}

纵观中医文献, 美白古方以活血法为主(Fang \& Song, 2012a; Fang \&Song, 2012b; Fang \& Song, 2013) 人体面部肤色在血气充足 的情况下才能表现红润的健康状态, 血量不足或血流不畅将导 致面色晦暗及色素沉着, 如常见的女性黄褐玟, 已有研究证明 其发生与血液乘度增加导致血液淤滞, 即皮肤微循环障碍有一 定关系(Tang \& Ou, 2008)。再者，血液循环为皮肤的成纤维细 胞、胶原蛋白等提供充足的氧分和营养物质; 有利于将细胞的 代谢产物和各种有害物质及时清除，保证了皮肤的新陈代谢。 同时，当皮肤微循环速度加快，在单位时间内通过皮肤血管的 红细胞数量增多, 红色的氧化血红蛋白也随之增多, 从而增加 皮肤的红色色素含量, 肤色就会变得红润白晳(Saikia,2006)。 血管是个内分泌组织, 其中的皮肤微血管内皮细胞 (human dermal microvascular endothelial cell, HDMEC) 可分泌很多细胞 因子。从血管内皮细胞分泌的细胞因子角度来看, 血管内皮细 胞可以合成血管活性物质NO及ET-1, 这些活性物质对血管的 舒缩功能与血液流动性有着不可替代的调节作用。其中, ET-1 具有强大的收缩血管的作用, 可调节局部血管的紧张度 ( Yan et al., 2000)。而根据前文所述, ET-1具有促进黑素合成的功效。 因此，可通过促进微循环的方式调控ET-1含量, 进而从血管旁 分泌途径实现对黑素的调控达到美白功效。

\section{Conclusion}

美白与皮肤的光学属性密切相关, 皮肤各层结构均能影响肤 色, 但目前美白功效添加剂的开发研究中仍主要以干扰黑素代 谢为主, 未能从整体观角度研究美白。且在黑素代谢的研究应 用中, 主要以抑制酪氨酸酶活性这条路径为主, 忽视了这条路 径的整体观，过度开发，导致了一系列安全风险。故我们在 实际开发应用时, 需遵循皮肤代谢规律, 从整体出发, 科学美 白, 健康一生。 


\section{References}

Abdel N. Differential effects on melanocyte growth and melanization of low vs. high calcium keratinocyteconditioned medium. British Journal of Dermatology, 140: 50-55, 1999.

Baroni A, Buommino E, De Gregorio V, Ruocco E, Ruocco V, Wolf R. Structure and function of the epidermis related to barrier properties. Clinics in Dermatology, 30: 257-262, 2012

Chen DS, Liu ZK. A survey of skin color detection. Chinese Journal of Computers, 29: 194-207, 2006.

Fang L, Zhao ZM. Research progress in the mechanism of skin aging. People's Military Surgeon, 60: 149-150, 2010.

Fang Y, Song P. Review on ancient traditional Chinese medicine external use prescriptions with skinwhitening, macula-removing, stagnationdissipating and scar-removing efficacy chapter one: characteristics of prescriptions and medicine before Tang dynasty. Chinese Journal of Dermatovenerology of Integrated Traditional and Western Medicine, 11: 334-336, 2012a.

Fang Y, Song P. Review on ancient traditional Chinese medicine external use prescriptions with skinwhitening, macula-removing, stagnationdissipating and scar-removing efficacy (chapter two): characteristics of prescriptions and medicine in Song-jin-yuan dynasty. Chinese Journal of Dermatovenerology of Integrated Traditional and Western Medicine, 11: 392-394, 2012 b.

Fang Y, Song P. Review on ancient traditional Chinese medicine external use prescriptions with skinwhitening, macula-removing, stagnationdissipating and scar-removing efficacy (chapter three): characteristics of prescriptions and medicine in the Ming-qing dynasty. Chinese Journal of Dermatovenerology of Integrated Traditional and Western Medicine, 12: 192-195, 2013.

Gillbro JM, Olsson MJ. The melanogenesis and mechanisms of skin-lightening agents-existing and new approaches. International Journal of Cosmetic Science, 33: 210-221, 2011.

Gu JM, Xu J, Chen LJ. The formation mechanism and countermeasures for skin showing dark yellow in the skin aging process. Chinese Journal of Aesthetic Medicine, 20: 2002-2004, 2011.

Gordon PR, Mansur CP, Gilchrest BA. Regulation of human melanocyte growth, dendricity and melanization by keratinocyte derived factors. Journal of Investigative Dermatology, 92: 565-572, 1989.

He L. Skin cares science. People's Medical Publishing House, Beijng, pp46-48, 2011.

Huang XF, Gu H, He L, Ma JB. Research progress of plant whitening agents. Chinese Journal of Dermatology, 46: 688-690, 2013.

Ha JH, Kang WH, Lee JO, Cho YK, Park SK, Lee SK, Cho HK. Clinical evaluation of the depigmenting effect of Glechoma hederacea extract by topical treatment for 8 weeks on UV-induced pigmentation in Asian skin. European Journal of Dermatology, 21: 218-222, 2011.

Kasamatsu S, Hachiya A, Shimotoyodome Y, Kameyama A, Miyauchi Y, Higuchi K, Fujimori T, Ohuchi A, Shibuya Y, Kitahara T. The inhibitory effect of a Platycodon root extract on ultraviolet B-induced pigmentation due to a decrease in kit expression. Journal of Natural Medicines, 68: 643-646, 2014.

Kim HJ, Yonezawa T, Teruya T, Woo JT, Cha BY. Nobiletin, a polymethoxy flavonoid, reduced endothelin-1 plus $\mathrm{SCF}$-induced pigmentation in human melanocytes. Photochemistry and Photobiology, 91: 379-386, 2015.

Kim JH, Sim GS, Lee DH, Lee GS, Lee BC, Pyo HB. New whitening agent from Pimpinella brachycarpa. Journal of the Society of Cosmetic Scientists of Korea, 33: 203-208, 2007.

Kobayashi A, Hachiya A, Ohuchi A, Kitahara T, Takema Y. Inhibitory mechanism of an extract of Althaea officinalis L. on endothelin-1-induced melanocyte activation. Biological and Pharmaceutical Bulletin, 25: 229-234, 2002.

Lu HG, Pearse A, Marks R. Racial differences of melanin content and morphology in surface corneocytes: a comparison between black, white and oriental skins. Chinese Journal of Dermatology, 33: 254-256, 2000 .

Lai JX, He CF, Dong YM. Research development on the mechanism of skin aging and anti-aging cosmetics. 
Chinese Journal of Aesthetic Medicine, 18: 12081212, 2009.

Niwano T, Terazawa S, Nakajima H, Wakabayashi Y, Imokawa G. Astaxanthin and withaferin a block paracrine cytokine interactions between UVB-exposed human keratinocytes and human melanocytes via the attenuation of endothelin-1 secretion and its downstream intracellular signaling. Cytokine, 73: 184-197, 2015.

Park SH, Lee BY, Han CS, Kim JG, Kim KT, Kim KH, Kim YH. Whitening effect of Angelica keiskei koidzumi extract by inhibition of endothelin-1 production and melanogenesis. Journal of the Society of Cosmetic Scientists of Korea, 34: 101-107, 2008.

Ramos-e-Silva M, Jacques Cd. Epidermal barrier function and systemic diseases. Clinics in Dermatology, 30: 277-279, 2012.

Saikia AP, Ryakala VK, Sharma P, Goswami P, Bora U. Ethnobotany of medicinal plants used by assamese people for various skin ailments and cosmetics. Journal of Ethnopharmacology, 106: 149-157, 2006.
Tang ZK, Ou YB. Literature analysis of nourishing yin and promoting blood flow method treat chloasma. Chinese Journal of Aesthetic Medicine, 17: 15461549, 2008.

Wang Y, Zhao Y, Liu L, Zhang L, Xiao H, Wu K, Xu $\mathrm{Y}, \mathrm{Hu} \mathrm{Y}, \mathrm{Fu} \mathrm{H}$, Cao W, et al. Inhibitory effects of imatinib mesylate on human epidermal melanocytes. Clinical and Experimental Dermatology, 39: 202208, 2014.

Yan YY, Zhao CF, Li SJ, Yu LJ. Mechanism of actions of skin-whitening cosmetics and its formulation development. China Surfactant Detergent \& Cosmetics, 39: 423-427, 2009.

Yue L, Yu XY, Zhang GG. Progress of herbal application in skin disease. Journal of Shenyang Pharmaceutical University, 55: 841-845, 2011.

Yan GF, He W, Liu RQ. Effect of vascular endothelial cells on fibroblast proliferation. Chinese Journal of Dermatology, 33: 29-31, 2000. 


\section{국문초록}

\section{과학적인 피부미백에 대한 전체론}

두탁, 리려, 동은묘*

북경공상대학, 중국 화장품공동혁신센터, 북경, 중국

목적: 본 논문의 목적은 피부색상에 영향을 주는 인자에 대해 전반적으로 분석하여, 건강한 피부 미백을 위한 새로운 방법을 제공 하는 것이다.

방법: 먼저 피부색상에 근본적인 영향을 주는 인자에 대해 논하였으며, 과학적으로 피부미백을 달성할 수 있는 여러 개발가능한 방법을 상세히 검토하였다. 마지막으로 메커니즘 수준에서 멜라닌 대사, 혈액순환, 진피 콜라겐 섬유 등 세가지 방면으로 건강하고 안전한 미백경로에 대해 분석하였다.

결과: 각질 형성세포와 연관된 사이토카인의 방출억제, 혈액순환 촉진 및 피부 콜라겐 섬유의 파괴를 억제하는 등 세가지 측면에서 건강한 피부미백경로를 탐색하였다.

결론: 피부 미백은 피부의 광학속성과 밀접한 상관관계를 갖고 있으므로 피부 각 층은 모두 피부색상에 영향을 준다. 그러므로 과학 적인 방법으로 피부미백을 달성하기 위해서는 피부 대사의 규칙을 따라야 한다.

핵심어: 전체론, 미백, 광학속성, 혈액미순환, 콜라겐 섬유 


\section{中文摘要}

\section{以整体观诠释科学美白}

杜卓, 李丽, 董银卯*

北京工商大学, 中国化妆品协同创新中心, 北京, 中国

目的: 本文旨在通过从整体观出发，全面剖析影响肤色的相关因素，为健康美白提供新思路。

方法: 首先从根源上探讨了影响肤色的相关因素，阐述了可拓展开发的美白路径，最后在机理层面上从黑素代谢、血液微循环、 真皮胶原纤维三个方面深度剖析了如何健康安全美白。

结果: 我们可通过抑制角质形成细胞相关细胞因子释放、促进血液微循环、抑制真皮层胶原纤维断裂黄化三个方面开拓健康美 白新路径。

结论: 美白与皮肤的光学属性密切相关, 皮肤各层结构均能影响肤色, 我们在实际开发应用时, 需遵循皮肤代谢规律, 从整体 出发, 科学美白, 健康一生。

关键词: 整体观, 美白, 光学属性, 血液微循环, 胶原纤维 